\title{
CASE REPORT: PATHOLOGICAL COMPLETE RESPONSE IN BREAST IN A PATIENT WITH METASTATIC BREAST CANCER TREATED WITH ANASTROZOLE
}

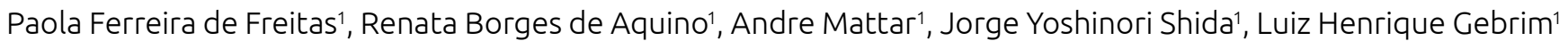
'Pérola Byington Hospital, Women's Reference Center - São Paulo (SP), Brazil.

Background: De novo metastatic breast cancer is seldomly encountered in patients visiting doctor's clinics for the first time. This article reports a case of cervical/submandibular metastatic breast cancer treated with neoadjuvant endocrine therapy (NET) with anastrozole (ANA). Case description: We herein report on a patient affected by HR+, HER2-, and metastatic breast cancer treated with NET that presented after 6 months with the possibility of breast-conserving surgery due to clinical and radiological complete response, revealing after surgery pathological complete response. Conclusion: Even for patients with metastatic luminal breast cancer, NET with an aromatase inhibitor, especially ANA, is a good option for postmenopausal women with fewer side effects and allowing for breast-conserving surgery with less morbidity when comparing with chemotherapy and yet yielding good results.

Keywords: Breast Cancer; Metastatic Breast Cancer; Neoadjuvant Endocrine Therapy; Anastrozole. 